\title{
Large Scale Process System Optimization
}

\author{
Milan Milivojevic ${ }^{1}$, Stanko Zerajic ${ }^{2}$ \\ ${ }^{1}$ Faculty of Technology and Metallurgy Belgrade University, Karnegijeva, Belgrade, Serbia \\ ${ }^{2}$ Faculty of Technology, University of Nis, Leskovac, B.Oslobodjenja, Leskovac, Serbia
}

Email address:

mmilan@tmf.bg.ac.rs (M. Milivojevic), zerajicstanko@gmail.com (S. Zerajic)

\section{To cite this article:}

Milan Milivojevic, Stanko Zerajic. Large Scale Process System Optimization. Science Research. Vol. 3, No. 3, 2015, pp. 58-65.

doi: $10.11648 /$ j.sr.20150303.13

\begin{abstract}
Optimization requires object function definition, constraints, and criteria. Complex optimization with more independent variables and more object functions has some diffulcuties. Simplified models often are very useful to represent the problem adequately over small ranges of the independent variables. Because of that structure representation methods were studied in this paper. Block diagrams, system streams, block element with system inputs and outputs were examined. In this paper an approach of complex optimization of production system by duals optimization was derived.
\end{abstract}

Keywords: Complex System, Structure, Representation, Manufacturing, Decomposition

\section{Introduction}

In most aspects of industrial life continual improvement is an important feature. Thus need to get the largest production from given raw materials, the greatest profit from a fixed investment, and so on [1]-[6]. Optimization is a formal presentation of these ideas.

Improvement can regarded from two view profits. Economic improvement provides an overall framework in which a given situation must be examined, since all problems must be considered within a financial structure. Many problems, however, may not be directly related to the company finances, and for this reason many improvements will be carried out on a technical basis.

In many cases it might be argued that use of formal optimization methods is worthwhile. Thus, for example, data may be inaccurate or the assumptions used in constructing a model for equipment design may be oversimplified. These situations are certainly found in practice, and therefore optimization results cannot be blindly accepted. In addition, it may be found that it is almost impossible to achieve the stated optimal conditions [7]-[10].

The aim of this paper was to seek out an adequate representation of the complex system optimization.

\section{System Representation}

The structure of most problems in optimization is amazingly similar. In order to design and operate any system or process, it is necessary to obtain a suitable model representing the system, to obtain solutions, to choose a suitable objective criterion which will guide any decisions, and to find a method of optimization.

The model may be one of two types. First, a mathematical model may be postulated to represent the process system, in which analytical relationships, together with appropriate restrictions, define the response of the process. Alternatively the process may already exist, and in the case the response to various input signals may be obtained by experimentation. A model developed from this type of experimental information is called a black-box or gray-box model, since it is not necessary to know the governing equations, but only the range of inputs to be selected. Sometimes, especially for more complex mathematical models, a system adequately represented by a mathematical model may be treated in the second manner, so that outputs such as profits or yields are determined numerically for a given set input values. These outputs are treated in the same way as black-box outputs.

Once the model has been defined and a solution is obtainable, optimization methods may be selected, assuming that suitable selection criteria are available and simulation is achieved. The criteria essentially determine the information required in the optimization. Thus an investigation of the optimum conditions yielding the most profit at the design stage necessitates finding the capital, the operating costs, and the income for the process.

In other hand, if a minimum cost were the only object, it would not be necessary to include the project income. 
Similarly, the capital and the design variables of an existing system would not be required. The optimization once a given problem has been set in the form of an objective function, determined either by numerical data from a black-box model or from mathematical functions, subject to various equality or inequality restrictions. It may be of many different forms, ranging from a straight ward comparison between two results to the most elegant of mathematical treatment.

The important feature is, however, the obtaining of an improved solution. It should be noted that optimization may not only modify the system variables, but in addition can alter the system itself. Thus one type of reactor might be replaced by another, a batch by a stirred tank flow reactor, and so on, or the arrangement of the flowsheet may be changed.

A realistic model is the most important factor in any optimization study. It is of little use to carry out improvement studies based on a model which does not adequately represent the existing or proposed system. Although the model will not be discussed, except as required to provide a basis for optimization problem, the importance of the choice of the model can not be emphasized to strongly. In the same vein, it should be noted that too complex a representation creates grave problems either in optimization or in model building. Such detail would, however, be required in the final stages of an engineering design. Simplified models often can be used to represent the problem adequately over small ranges of the independent variables.

\section{The Structure of System Optimization}

The optimization of any system, process, or project cannot be accomplished without first assembling the appropriate information in a suitable form. The step of definition for optimization is therefore a critical one for problem considering.

The objective function must ultimately, be expressed in terms of those system variables which can be manipulated or which are controlled by an external agency. The task can often be facilitated by preparing an adequate representation of the system and by understanding the nature of the component parts of the system.

There are a number of ways in which the important topological features of a system can be represented. Generally, a block diagram is used, but with computer calculations other forms are sometimes preferable.

The structure of the system is taken to mean the arrangement and interrelationship of the various units or components constituting the total system.

The choice of an appropriate objective is not straightward, although it is usually dictated by company policy.

\subsection{Optimization and Profit Objective Functions}

Most objectives will be normally minimized or maximized as appropriate. If investment is included, however, misleading results might be obtained, unless it is realized that both absolute and marginal rates of return must be examined. Thus, even though the absolute rate of return is acceptable, operating in the region $I_{1} \leq I \leq I_{5}$, as shown in Fig. 1, the need for an adequate incremental or marginal rate of return greater than some specified minimum value $\alpha$, obtainable from an alternative investment, makes it more profitable to operate the process below $I_{3}$. Any additional capital should then be invested in the alternative investment, yielding the total profit-investment relation, as given by curve ABCD.

The procedures show the operation under maximum profit is less advantageous. Normal operation would preferably be in the region $A B C$, with $\mathrm{B}$ yielding the maximum profitability (Fig. 1).

Such as $P I$ envelope is the upper limit of a set of $P-I$ results determined for various alternative investments in different systems or in different arrangements within a given system. Such alternatives exist, for example, in the case when they represent different chemical processes or various flowsheet arrangements. However, even if a single project is being evaluated, the results can be compared with the alternative offered by making no investment and leaving the capital in a bank or similar source of income.

Since, the sale income is essentially a variable, dependent upon external agencies, such as competition, markets, and advertising; it is often preferable not to include this, but to use the net realization criterion in project evaluation.

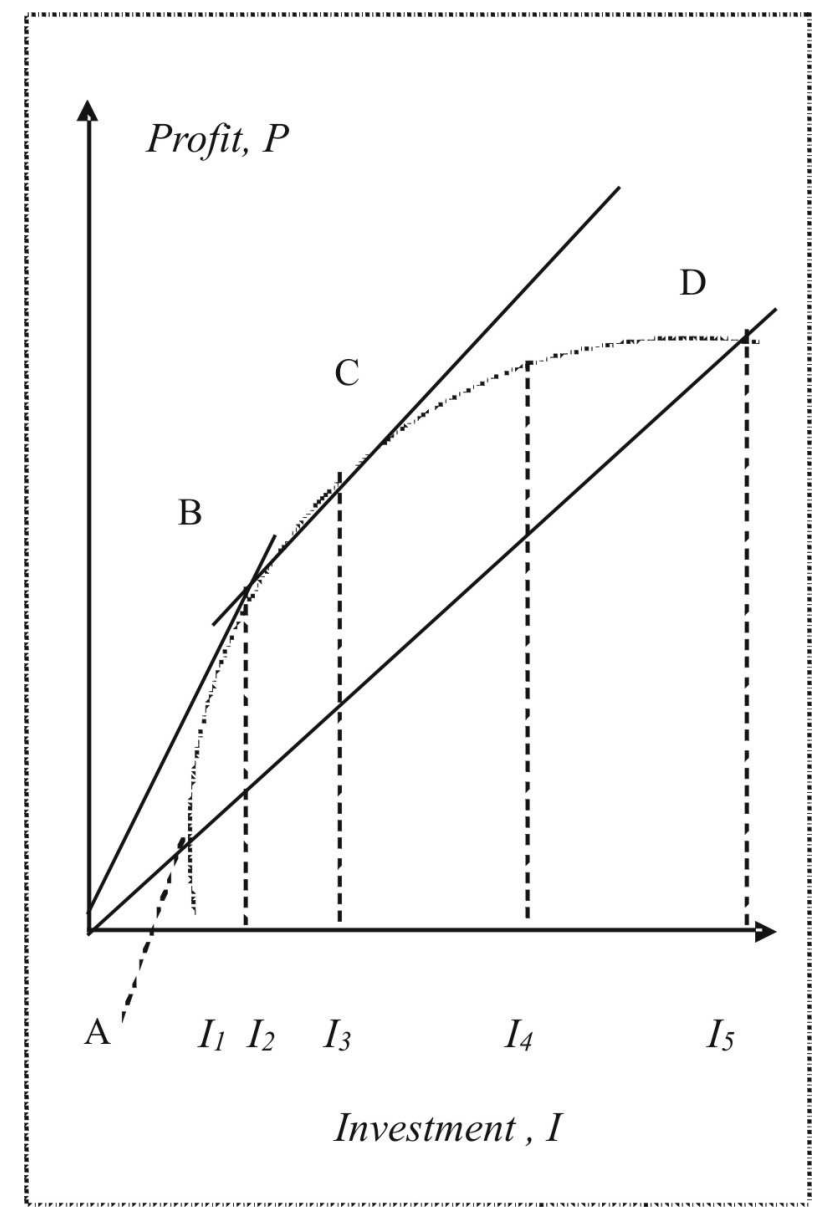

Fig. 1. Profit versus investment.

This allows the maximum competitive position to be found, and the sales income to be determined separately. 
While most objectives are to be maximized or minimized, there are situations in which expected values must be optimized when the system is stochastic in nature. Uncertainty, due to competition can also require such approaches as the minimization of the maximum loss.

\subsection{Representation of Structure}

The block diagrams, system streams, the block element and mathematical representation of system variables are various methods for system representation.

Block diagram, generally, is the most often used (Fig.2), but other forms are sometimes preferable.

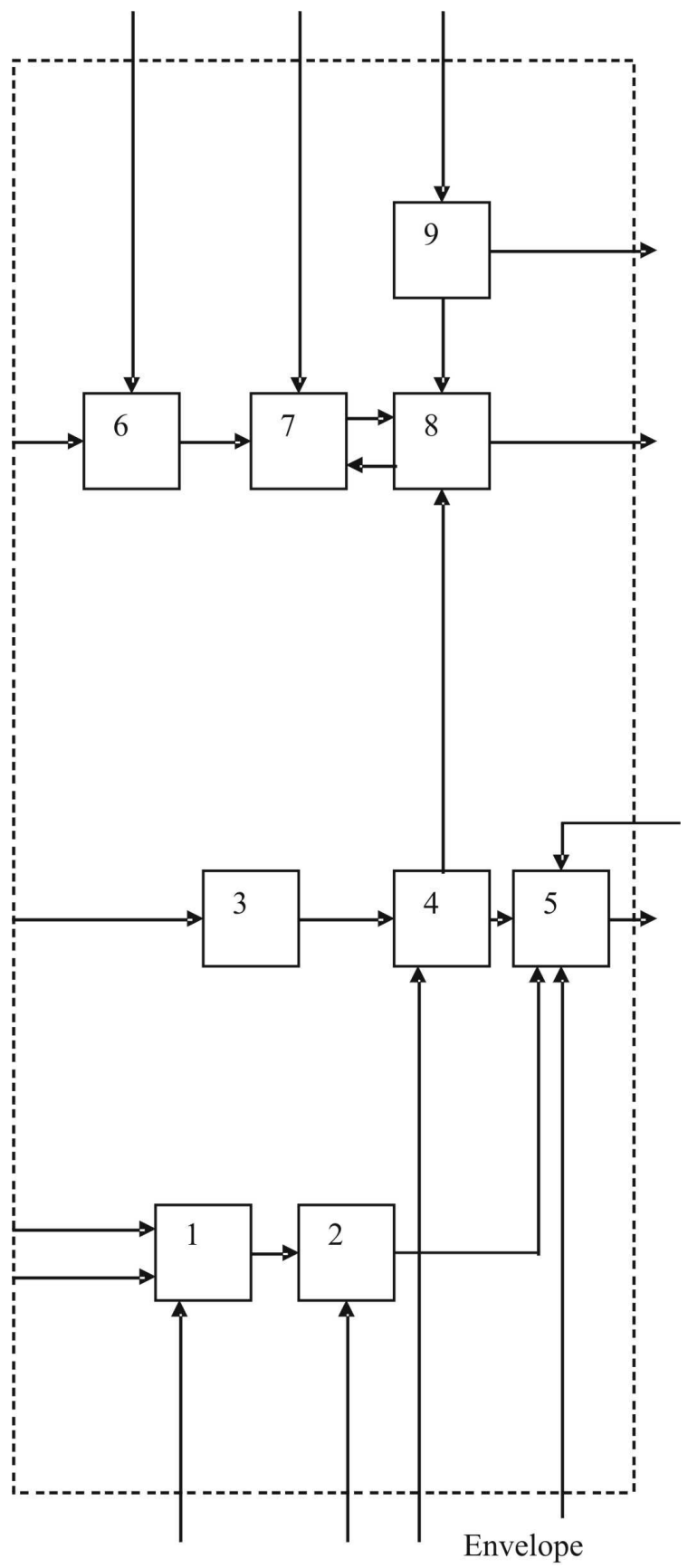

Fig. 2. The system structure.
The structure of the system is taken to mean the arrangement and interrelationship of the various units or components constituting the total system. This can be represented by a block diagram such as is presented in Fig. 2.

Each block might represent such items as an oil refinery, a distillation column, or tray within a distillation column, depending upon the nature of the particular problem and the level of optimization. . The whole diagram represents the system. The interconnecting lines show the system streams, which could, for example, be fluid streams, lines of information communication, heat flow, or even the flow of money into and out of a business operation.

While such diagrams can represent the spatial arrangement of equipment linked by physical streams, they can also be used to show time variations, which each block representing a physical unit at a given stage in time. Examples include a batch reactor in which the heat input is changed after finite time intervals and a plant in which money is spent annually over a period of years for such items as planned maintenance.

These convenient flow diagrams or block diagrams provide information about the lines of communication between various parts of the system. This is an essential step in the construction of an objective function.

The diagrammatic representation, conceptual model, as long as there is some human interpretation during calculation. Different forms, however must be considered is convenient even for complicated systems

Streams system is very useful of system representation. Three types of system streams can be distinguished. Those streams which enter the system from the outside are called inputs. Simularly, outputs streams are those leaving a unit, conveying product from the system. The interlinking streams both orginate and terminate in the system, connecting the various units. Examples of each type are shown in Fig. 3.

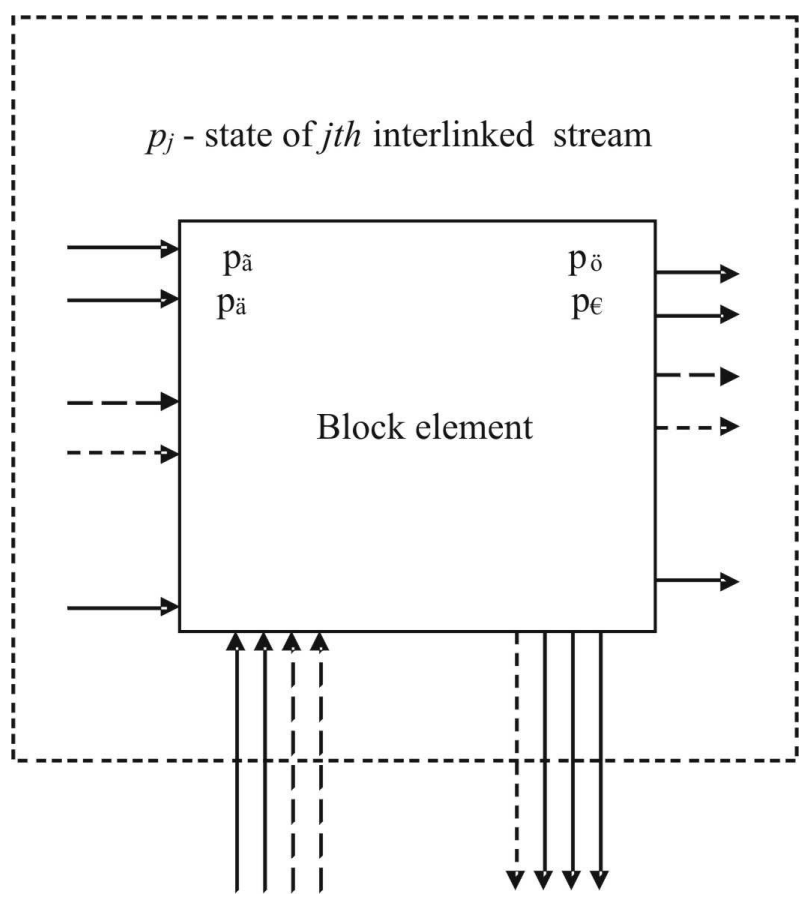

Fig. 3. Block element with system inputs and outputs. 
To evalute the objective function, all these streams must be defined. This definition can be achieved by specifzing several variables associated with each stream. Thus, in the analysis of a chemical reactor, the feed stream might be specified. This can be done only if the composition, temperature, and flow rate of the stream are all given. These quantities, when given, specify the state of that stream. In other cases a single variable might be sufficient to represent the conditions within a stream, as in the case of the flow of investment capital or the rate of fresh catalyst addition to a reaction system.

The state of $j t h$ interlinking stream may be denoted by the vector $\bar{p}_{j}$. Here, $\bar{p}_{j}$ consists of a list $\left(p_{1}, p_{2}, \ldots \ldots \ldots . . ., p_{j}\right)$ of the $P_{j}$ system variables required to specify the state of the $j$ th process stream. A component of $\bar{p}_{j}$, say $p_{j, i}$ might, for eample, be a flow rate or a temperature. Here the vector notation is metely a convenience indicating the several variables which may be required to determine the state of a single process stream. The state of given stream is completely specified when all the system variables contributing to the objective function are given. Thus, in the design of heat exchanger, a stream of material may be specified by its temperature and flow rate, but its composition is not particulaly important. However, in the design of a chemical reactor, the stream composition would be one of the most significant quantities. Similarly, the state of the $k t h$ input stream can be represented by $\bar{q}_{k}$, and the lth output stream by $\bar{r}_{l}$. Such a notation may be used directly or replaced in appropriate cases by mnemonic symbols ( $\mathrm{T}$ for temperature, etc.) or a general variable $x_{i}$.

The block element can not proceed until the behavior of each of the system units, represented by block, is successfully modeled. The existence of such a technical design implies that if given the states of all the entering interlinking streams $\bar{p}_{j}$ and the inputs all $\bar{q}_{k}$, the outputs $\bar{r}_{l}$ are determined either exactly or in terms of stochastic functions.

System variables and objective connections state model can complete represent the system. The model has to be found in the form of a transformation equation which express the outputs in terms of the inputs. Thus, for a deterministic system, can write that:

$$
\begin{aligned}
& \bar{p}_{\Phi}=\bar{T}_{\Phi}\left(\bar{q}_{\alpha}, \bar{q}_{\beta}, \ldots . ., \bar{p}_{\gamma}, \bar{p}_{\delta}, \ldots .\right) \\
& \bar{p}_{\varepsilon}=\bar{T}_{\varepsilon}\left(\bar{q}_{\alpha}, \bar{q}_{\beta}, \ldots . ., \bar{p}_{\gamma}, \bar{p}_{\delta}, \ldots .\right) \\
& \bar{r}_{\theta}=\bar{T}_{\theta}\left(\bar{q}_{\alpha}, \bar{q}_{\beta}, \ldots ., \bar{p}_{\gamma}, \bar{p}_{\delta}, \ldots .\right) \\
& \bar{r}_{\psi}=\bar{T}_{\psi}\left(\bar{q}_{\alpha}, \bar{q}_{\beta}, \ldots . ., \bar{p}_{\gamma}, \bar{p}_{\delta}, \ldots .\right)
\end{aligned}
$$

where $\bar{p}$ referes to state, $\bar{q}$ is inputs, $\bar{r}$ is outputs, and $\bar{T}$ is transformation.

It may sometimes be necessary or desirable to fix the output of a particular block at a given value. In this case it might be convenient if one of the inputs could be expressed in terms of the other inputs and the specified output.

\section{Manufacturing System Optimization}

Manufacturing developing involves deciding what operations are to be involved and in what sequence they are to be performed.

Chemical process engineering today is concerned with the understanding and development of systematic procedures for design and optimal operation and control of the chemical process systems, ranging from nano-, micro-, macro-systems to industrial scale. This work motivated by the need to provide a more flexible than existing approaches, framework for changes and optimization in chemical process engineering in particular, and for predicting the behavior of production systems in general.

The structural model is generated according to several levels decomposition of the process unit into phases and components. The decomposition begin with system definition. Definition includes all components, input and output attributes, state variables, behavior rules, and initial conditions [8].

In manufacturing system optimization need to satisfy minimization and maximization object functions. The manufacturing system optimization requires conflict resolution between minimum cost and maximum product quality and capacity.

These systems require duals in optimization.

Let consider concerning with optimization of an objective function, $y(x)$ by selecting a suitable set of variables $\bar{x}$, which satisfy all the imposed constraints. A minimization problem can be expressed as:

$$
M=\min { }_{x} y(x)
$$

where $\bar{x}$ must be satisfy all imposed restrictions. Let suppose that there exists a second function $W(v)$ which has the same value $M$ at its maximum, so that,

$$
M=\max \underset{x}{ } y(x)
$$

with $\bar{v}$ satisfaying certain restrictions. Then can state that: $y(x)$ for all permited $x \geq M \geq W(v)$ for all permited $v$.

Therefore, $y(x)$ provides an upper bound on the particular value of $M$ and $W(v)$ is a lower bound. The two problems defined by eqs(2)-(3) are dual problems, with in general, one specified as the primat problem and the other its dual. Here the function $W(v)$ is defined as the dual of the primal problem.

This concept of duality in optimization requires duals can be constructed. If the dual to an optimization problem can be 
formulated, important computational advantages can often be gained. For example, upper and lower limits to the true value of the optimal objective function are given immidiately by approximate solutions from inequalities, eq.(4). In some cases the dual might be a simpler problem than the primal.

\section{Optimization Objectives and Restriction}

The optimization of any system, process, or project cannot be accomplished without first assembling the appropriate information in a suitable form. The step of definition for optimization is therefore a critical one for problem considering. Almost any problem in the design, operation, and analysis of manufacturing plants and industrial processes, and any associated problem such as production scheduling, can be reduced in the final analysis to the problem of determining the largest or smallest value of a function of several variables.

Since optimization is the collective process of finding the set of conditions required to achieve the best result from a given situation, it follows that the techniques of optimization must be brought to bear on every task of practical importance. The subject, seemingly simple, is in fact a complex one, and can best be mastered by viewing the components of an optimization study before carrying out the complete task. Before defining these components it is perhaps instructive to note the scope of the problem. It will be seen on careful analysis that the optimization of the problem will itself fall into the realm of optimization.

Irrespective of the application, several features can be distinguished in most optimization studies.

Any problem in an optimization analysis will have as its objective the improvement of the systems. It should be fairly obvious that, in order to improve any system, it is essential that at least one solution be obtainable for that system. In other words, by defining the inputs to a system, can find the resulting output. If this is not possible, can not design or operate or control the system, far less optimize it.

No single answer is normally found to any problem, and it is therefore necessary to choose the best solution for a given problem from the multitude of possible solutions. How can this be achieved? First, it is necessary to define the objective of the study. This may vary from one problem to another, but for industrial applications it might be either economic or technological. Such economic aims as maximum profit or minimum costs are common, while possible technical objectives might include the largest yield of a particular product from a reactor or the smallest surface area for a given volume of vessel. Normally, however, most industrial optimization has to be carried out within an economic framework.

It should also be noted that not all problems are capable of quantitative analysis, although all have the general objective of creating a better set of conditions to achieve the best possible results. In general, it is necessary to have a quantitative measure in order to provide a comparative basis, and thus all problems must be formulated so as to present all their information in a quantitative form, even though relative numbers have to be allocated in an empirical manner to evaluate a set of conditions. This quantitative form is the objective function by which the criterion of selection is related to the system variables.

What means absolute best? Although optimization studies may be required to achieve the best result from a given situation, meaning the absolute best, this may not always be attainable due to the imposition of restrictions.

If the problem is unrestricted, may take an example the case of a batch extraction unit in which the largest amount of extraction will be attained with an infinite amount of solvent. Then has an absolute best in the sense of the total amount of solute which can possibly be removed. When restrictions are imposed as on the availability of solvent, a smaller amount will be extracted. It can still, however, regard this latter condition as an optimum, but it is not an absolute optimum, only one relative to the restriction.

\section{Levels of Optimization}

Considered some salient features, having seen the types of problem encountered in optimization studies, let now look to the general problem of plant design and operation, a problem which need not be specifically defined at this stage, but which will be illustrated by general reference to chemical plant.

In the manufacturing industries the project may be of varying complexity as a combination or complex of factories, works, or companies, for example, individual oil refineries within an oil company, which itself may form a component of a group of companies. Then, project may be a combination of unit processes, as in a chemical works or oil refinery in which a network of individual plants using interconnected feeds and products yields a range of products from a given range of raw materials. Also, project may be a combination of components, such as distillation towers, filters, heat exchangers, reactors, and the like, arranged in a unit process, such as an acetic acid plant.

Finally, project may be a specific component, such as a heat exchanger.

Whatever the level of complexity, the project may, in addition, be analyzed from the point of view of design, operation, or control. For example, at design, at the third level above, project of a combination of component, the available capital might be used to build a plant for the production of a salable chemical. Here the sale may be within the company or on the open market, but the same arguments will apply to both cases, since, if a chemical can not be produced cheaply enough within a company, it will be bought outside, unless there are other reasons preventing this, such as desire for control of sources, quality, etc. In this plant design some or all of the points such as 1- design and management level, 2-new projects and plants, 3-process and flowsheeting, 4-equipment specification, and 5-operating conditions can be involved.

Design and management level involves evaluation of a given project, comparison between projects, and capital 
allocation among projects.

New project or plant involves choice of product, choice of plant capacity, choice of chemical route, choice of raw materials, choice of site location.

Process involves choice of process, choice of process flowsheet, arrangement of flowsheet etc.

Equipment specification involves selection of type of equipment for a given flowsheet duty, choice of materials of construction, sizing, of equipment units and layout.

Operating conditions involve time dependent and time independent process units, control systems, maintenance and equipment replacement policies, scheduling of production, allocation of raw materials, storage inventories for raw materials, intermediates, and products, shipping, transportation, and distribution.

Optimization problems can be found at all these levels. It will be noted that the scope of the optimization problem can be the entire business enterprise, a process, a single operation, a single component in that operation or any intermediate stage between these. The depth of the study may involve generalization indicating only trends, or it may examine the minute detail, depending upon the use of which the results will be put, the availability of accurate data, and the time available in which to carry out the study.

While the technology may be chemical, business, or any other type, optimization methods can be applied to any individual stage. For the best results, any optimization study ought to be included from the earliest period in the design analysis. It should thus be taken into account from the initiation of a project and be regarded as an integral part of the design process, and not treated as a series of small isolated maximization or minimization problems. Actually, improvements can be carried out at any isolated stage in the design, as in the simple illustrations quoted previously, but if this is done, it does not follow that the optimal result for the integrated process will be found.

If noted that the complete project design and optimization as being the highest level. Correspondingly, the problem of design and operation of the chemical plant can be entered as successively lower levels, but after entering at a special level, for example, at the discussion of equipment size, need to consider and optimize all successive stages in order to achieve the optimum conditions.

The ultimate in design technique, and hence in optimization, is the synthesis of the complete reaction system, with the optimal control, operation, and choice of equipment having been made and the items arranged in the most suitable choice of flowsheet and chemical route to the desired product, all this being carried out as an integrated scheme. The degree of complexity of this synthesis is associated with the level of the design and optimization attempted, being most complex at the highest levels. In general, it may therefore be stated that must solve the problem of choosing a set of design and operating variables in such a way as to optimize some suitable criterion of selection while still satisfying all the constraints of the type indicated. In its complete generally, this problem is often so complex that it becomes impossible to tackle, either because sufficient information is not available to allow its formulation in precise terms or simply because of the enormous number of variables and constraints to be considered, often as nonlinear functions. Indeed, it might be said that the aim of engineering design studies is to put the designer in possession of all the necessary physical, chemical, mechanical, and economics data required to formulate such a problem with the full precision and confidence needed, whether the situation is at the design or the operation stage in a plant or process.

On much less ambitious scale than that just outlined, it has proved possible to solve general problems of this type by restricting their scope and by making a number of simplifying assumptions. The present state of optimization theory and practice application is such that the most complex process, that of the complete project, has not yet been handled in detail. Optimization of a chosen flowsheet and comparison of this with other flowsheets is the most complex type of situation considered as yet. The design of individual items of process equipment, such as converters, distillation columns, and heat exchangers, and the numerical optimization of complex mathematical functions are the two main areas in which optimization is at present most effective.

In order even partially to achieve these aims, engineers need to familiarize themselves with the various available methods of optimization.

\section{Methods for Optimization}

The necessity of determining a maximum or minimum in optimization might lead one to think of the elementary differential calculus approach to the problem of finding a stationary value of the function by considering the variation of the independent variables under the control of the designer or operator. However, in most realistic problems, the situation is normally complicated, due to the shape of a valid search region created by the system restrictions, the complexity and behavior of the functions under examination, and the possibility of certain functions being noncontinuous or nondifferentiable in the range of interest.

The general problem of finding the largest or smallest value of a function of one or many variables, when the function may be linear or nonlinear, discrete valued or continuous, exact or stochastic, restricted or unconstrained, is a very difficult one, and is still under development.

For this reason many types of optimization methods have been suggested, usually with a particular group of applications.

In general, however, the optimum can exist only at one or more of the following conditions, since it can not exist elsewhere, a stationary point, a discontinuity in the gradient or the function, or a boundary.

Should be noted that these points do not all need to be extreme values, and once has found their locations, it must find out if the corresponding objective function is in fact an extreme value. The search has now been reduced to a search for the location of these types of situation and a subsequent comparison of function values at these types of situation and a 
subsequent comparison of function values at these locations. Since, can not know in advance at which type of position the global optimum will lie, except in a few well behaved cases, it is necessary to examines all three possibilities, and any search must examine both the interior of the permitted region and its boundary.

The functions of examination can be of different forms. For example, in a multivariable system with many n-dimensional mountain peaks, the search for a maximum entails the search for the stationary values, as well as examination of other locations, a search which can sometimes be done analytically. Indeed, the analytical approach is the only one which will yield information on all the stationary point locations within the region. These methods will require assumptions as to the continuity of the function and its derivatives. The presence of discontinuities can be handled by the use of additional restrictions at each discontinuity.

If analytical methods are not convenient, as when a black-box model is producing the technical design data or when the expressions are too complex for convenient mathematical analysis, numerical search methods are required.This is by far the commonest approach, the search being again for a global optimum chosen from the local optima at stationary point, discontinuities, boundaries. The numerical approach can be linkened to starting off in a mountain vallwey, but blinndfolded. The search will involve the climb to the highest peak, but this must be achieved by repeated test moves. Once the local peak has been attained, there is no way of ascertaining by that search whether or not it is the highest peak of the whole mountain range except by starting from a new base location and again looking for another peak.

The only rigorous method of determining the global optimum, and the only way of handling discontunuities, is by direct comparison of the function values at the various local optimum, once these have been achieved.

\section{General Procedure for Optimization}

Since the optimization problem may usually be written in some mathematical form, it follows that the actual physical situation need be taken into account only when constructing the model. Several features may be distinguished which allow a common approach to be formulated, irrespective of the particular application investigated. These common features include distinguishing criteria, the need for a mathematical relationship linking these criteria to the set of system variables, i.e., a model, the requirement that certain of the system variables be controllable, the existence of constraints limiting the range of variation of some or all of the variables, and the importance of being able to calculate a first solution.

The general objective in optimization is to choose a set of values of the independent variables, subject to various restrictions which will produce the desired optimum response for the particular problem under examination. A general approach or procedure can be described as follows in Fig. 4.

Each of these steps needs to discuss in more detail for the problems in the field of applications. All these problems have the feature that, while at least one solution exists, there are usually an infinite number of solutions possible.

The aim of optimization is the selection, out of the multiplicity of potential solutions, of that solution which is the best with respect to some well defined criterion. The choose of this criterion, the objective, is therefore an essential step in any study.

\section{Conclusions}

In this paper method for information extraction, which required in the optimization was developed. The optimization problem has been set in the form of an objective function, determined by numerical data from a black-box model and from mathematical functions.

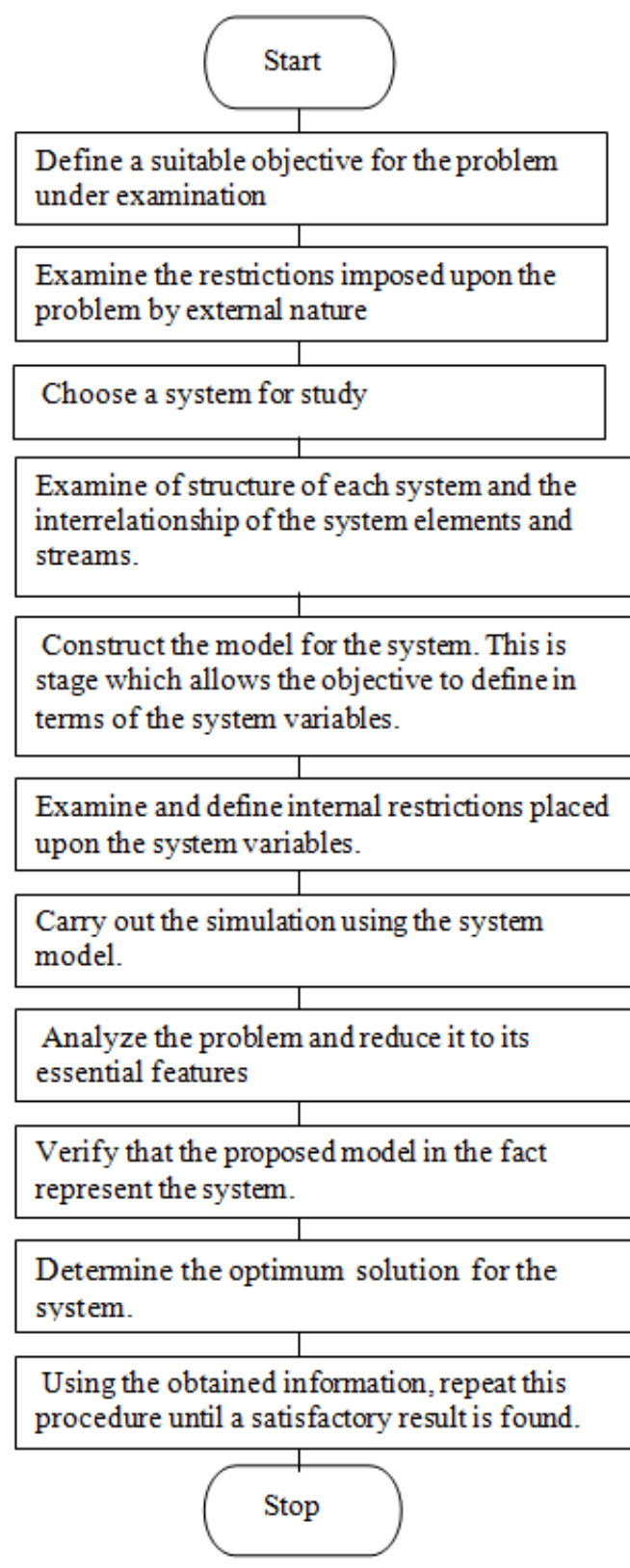

Fig. 4. Procedure of the complex system optimization. 
Profit-investment relation was considered. Normal operation is preferably in the region, with yielding the maximum profitability.

An approach to manufacturing system optimization is developed. The concept of diuality was considered.

Various levels of optimization were studied. Design and management level involves evaluation of a given project, comparison between projects, and capital allocation among projects. New project or plant involves choice of product, choice of plant capacity, choice of chemical route, choice of raw materials, choice of site location. Process involves choice of process, choice of process flowsheet, arrangement of flowsheet etc. Equipment specification involves selection of type of equipment for a given flowsheet duty, choice of materials of construction, sizing, of equipment units and layout. Operating conditions involve time dependent and time independent process units, control systems, maintenance and equipment replacement policies, scheduling of production, allocation of raw materials, storage inventories for raw materials, intermediates, and products, shipping, transportation, and distribution.

\section{Notation}

$$
\begin{aligned}
& \mathrm{M} \text { - extreme } \\
& p_{j} \text { - state of } j t h \text { interlinked stream } \\
& \mathrm{q} \text { - input } \\
& \mathrm{r} \text { - output } \\
& \mathrm{T} \text { - transformation. }
\end{aligned}
$$

\section{References}

[1] J.Savkovic-Stevanovic, An integrated optimization method in plant design, Proceedings of the 2nd Conference on Modeling and Simulation, pp.43-51, Belgrade 28-29 September, 2011, ISBN 978-86-911011-6-9.
[2] J.Savkovic-Stevanovic (1995) Optimization, chapter 9 in the book Process modeling and simulation, Faculty of Technology and Metallurgy Belgrade University, Belgrade, 1995.

[3] M.Vico-Stevanovic,J. Savkovic-Stevanovic, S.Jorgačevic, S. Joksimovic, Optimization of the Substrate in the Fed - Batch Fermentation of Antibiotics, CHISA90- Inter. Congress on Chemical Process Engineering and Equipment, paper A7.1, p.10, Praha, August 26-31, 1990.

[4] M.Vico-Stevanovic, J. Savkovic-Stevanovic ,S. Jorgačevic, S. Joksimovic , Optimization of the Supstrate Feed Rate for Fed-Batch Fermentation, IES'90-4 ${ }^{\text {th }}$ Seminar and Symposium on Informati on and Expert Systems in the Process Industries, p.2.13-2.27, Beograd, Oct., 4-5, 1990.

[5] S.Žerajic, J.Savković-Stevanovic, Bioprocess parametric optimization, CHISA2010 - Inter. Congress of Chem. Eng. and Process Engineering, CdROM, pages 9, Prague, August 29-Sept. 2, 2010.

[6] S.Zerajic, J.Savkovic-Stevanovic, Process parameters optimization, Comput. Ecol. Eng. vol.6(1)(2010)23-29, ISSN 1452-0729.

[7] D.Brzic, T.Kaludjerovic, J.Djurovic, Process systems optimization, Process systems in Chemical Engineering, Organizer: Milivojević M., The 2014 Chemical Engineering and Materials, Venice, Italy, March 15-17, 2014.

[8] J.Savkovic-Stevanovic, Process Engineering Intelligent Systems, RAJ, Memphis, 1999.

[9] G.P.Walsh, Methods of optimization, John Wiley \&Sons, 1975.

[10] V.G.Jenson, G.V.Jeffreys, Mathematical methods in chemical engineering, Academic Press, London, 1977, 